\title{
Non-Conforming Still's Disease With Low Ferritin and No Skin Rash: A Case Report
}

Dawlat Khan ${ }^{1}$, Muhammad Umar Saddique ${ }^{1}$, Muhammad B. Jamshaid ${ }^{2}$, Zohaib Yousaf ${ }^{1,} 3$, Mohamed A. Yassin ${ }^{4}$

1. Internal Medicine, Hamad Medical Corporation, Doha, QAT 2. Internal Medicine, Hamad General Hospital, Doha, QAT 3. Clinical Research, Dresden International University, Dresden, DEU 4. Hematology and Oncology, Hamad General Hospital, Doha, QAT

Corresponding author: Dawlat Khan, drhafiz2012@gmail.com

\begin{abstract}
Adult Still's disease (ASD) is an inflammatory disorder with an unclear etiology. It is a rare disease that was first described more than a century ago. Its common characteristics are daily fevers, arthritis, and skin rash. ASD is diagnosed after excluding infections, malignancies, and connective tissue diseases. It has a female predominance. Classic skin rash and high serum ferritin levels are commonly associated with this condition and help in the diagnosis. Due to a lack of pathognomic clinical and laboratory features, a valid diagnostic criterion, the Yamaguchi criteria, is generally used for the diagnosis. The disease has a good prognosis with appropriate treatment.
\end{abstract}

We present the case of a young gentleman who presented with fever, anemia, and leucocytosis; however, his serum ferritin levels were normal, and there was no typical salmon-colored skin rash. Hyperferritinemia developed later in the disease course, leading to a diagnosis of ASD.

Categories: Internal Medicine, Rheumatology

Keywords: still disease, high ferritin, fuo, puo

\section{Introduction}

Adult Still's disease (ASD) is a rare chronic systemic inflammatory disorder. Its annual incidence is 0.16 cases per 100,000 people, with a female-to-male ratio of 3:2 [1,2]. Initially described in children by George F. Still, in 1897, it remained overlooked until it was discussed again by Bywaters in 1971 [3]. It has a bimodal distribution with peaks in the age groups of 15-25 years and 36-46 years. The proposed etiology is multifactorial involving genetic factors, human leukocyte antigen (HLA), with possible infections and malignancies acting as triggers [4]. The classical presentation includes daily recurring fever (quotidian fever) or double quotidian fever (two fever spikes a day), accompanied by a salmon-pink maculopapular eruption overlapping with fever. Other signs that have been reported include arthralgia or arthritis, sore throat,

Received 05/05/2020

Review began 05/17/2020 Review ended 07/04/2020 Published 07/13/2020

\section{() Copyright 2020}

Khan et al. This is an open access article distributed under the terms of the Creative Commons Attribution License CC-BY 4.0., which permits unrestricted use, distribution, and reproduction in any medium, provided the original author and source are credited. weight loss, lymphadenopathy, and hepatosplenomegaly. Hyperferritinemia is very common, but its absence does not exclude ASD. It is a diagnosis of exclusion. Diagnosis is generally made after ruling out infections, malignancies, and connective tissue diseases by using the Yamaguchi or Fautrel criteria [5]. The prognosis is usually favorable with timely and appropriate therapy. In this report, we discuss an unusual case of ASD in a patient who presented without a skin rash and showed a delayed presentation of hyperferritinemia.

\section{Case Presentation}

An 18-year-old, previously healthy gentleman presented with a three-week history of high-grade fever, dry cough, throat pain, and arthralgia involving knees, ankles, and elbows. The fever was intermittent, with one episode per day and the temperature rising as high as $39^{\circ} \mathrm{C}$, which was relieved by acetaminophen. He had a history of unintentional weight loss and fatigue. Before presenting to the hospital, he had received a sevenday course of empiric antibiotics for suspected bacterial pharyngitis with no improvement. He denied any cough, hemoptysis, night sweats, rash, and gastrointestinal or urinary symptoms. He also denied any history of sick contacts, close tuberculosis (TB) contacts, and recent travel. There was no history of recurrent fever or joint pains in his family.

Clinical examination revealed a conscious, oriented, and hemodynamically stable patient with a temperature of $39.2^{\circ} \mathrm{C}$. He had mild pallor but not icteric, and small painless, soft, and mobile cervical lymph nodes were palpable. The rest of the examination including ankle and knee joints were unremarkable with no signs of inflammation.

He had microcytic anemia, marked neutrophilic predominant leukocytosis, high C-reactive protein (CRP), mild transaminitis, and normal lactate level. The blood and urine cultures taken during fever spikes were sterile. He had a negative malaria test and negative serology for brucellosis and mycoplasma. Additionally, viral hepatitis serology and respiratory viral panel, cytomegalovirus (CMV), Epstein-Barr virus (EBV), and 


\section{Cureus}

adenoviral polymerase chain reaction (PCR) were all negative, He also had a twice-negative sputum acidfast bacillus (AFB) stain and sputum TB PCR. The autoimmune workup was also normal (Tables 1, 2).

\begin{tabular}{|c|c|c|c|c|}
\hline Lab parameters & At admission & Fourth week & Eighth week & Reference range \\
\hline Hemoglobin & 10.4 & 10.7 & 12.4 & 13-17 \\
\hline White blood cell count & 28,000 & 25,000 & 10.5 & $4-10 \times 10^{3 / \mu L}$ \\
\hline Neutrophils & $87 \%$ & $81 \%$ & $40 \%$ & $40-60 \%$ \\
\hline Platelets & 459 & 367 & 300 & $150-400 \times 10^{3 / / \mu L}$ \\
\hline ALT & 89 & 53 & 13 & $0-40 \mathrm{U} / \mathrm{L}$ \\
\hline AST & 62 & 51 & 20 & $0-37 \mathrm{U} / \mathrm{L}$ \\
\hline Alkaline phosphatase & 104 & 110 & 86 & 40-120 U/L \\
\hline Total bilirubin & 5 & --- & 12 & 4-24 mmo//L \\
\hline Creatinine & 53 & 63 & 70 & 70-115 mmo//L \\
\hline Sodium & 135 & 141 & 143 & $135-145 \mathrm{mmol} / \mathrm{L}$ \\
\hline Haptoglobin & 657 & -- & $-\cdots$ & $30-200 \mathrm{mg} / \mathrm{dl}$ \\
\hline LDH & 328 & -- & -- & 105-235 U/L \\
\hline CRP & 279 & 150 & 14 & $0-5 \mathrm{mg} / \mathrm{L}$ \\
\hline Procalcitonin & 0.72 & $-\cdots$ & -- & $0.5 \mathrm{ng} / \mathrm{mL}$ \\
\hline Serum iron & 7.50 & -- & 7.50 & 4.80-24.7 mmo//L \\
\hline Serum ferritin & 35 & $>7,000$ & 601 & $30-400 \mathrm{mcg} / \mathrm{L}$ \\
\hline TIBC & 45 & - & 40 & 40-80 mmo/L \\
\hline Lactic acid & 1.1 & - & $-\cdots$ & $0.5-1.6 \mathrm{mmol} / \mathrm{L}$ \\
\hline Triglyceride & 2.2 & $\ldots$ & 0.79 & $<1.7 \mathrm{mmol} / \mathrm{L}$ \\
\hline
\end{tabular}

\section{TABLE 1: Hematological and biochemical investigations}

ALT: alanine transaminase; AST: aminotransferase; LDH: lactate dehydrogenase; CRP: C-reactive protein; TIBC: total iron-binding capacity 


\section{Cureus}

Test

Sputum AFB and TB-PCR (two sets)

QuantiFERON TB

Negative

Brucellosis (lgM/lgG)

Mycoplasma pneumoniae serology

Negative

Blood and urine culture

Negative

No growth

Hepatitis B and C

HIV (ELISA)

Blood CMV/EBV/adenoviral PCR

Rheumatoid factor

Anti-CCP

ANA

Anti-dsDNA

Negative

Negative

Negative

Normal

Negative

\section{TABLE 2: Bacteriology and autoimmune workup}

AFB: acid-fast bacillus; TB: tuberculosis; PCR: polymerase chain reaction; HIV: human immunodeficiency virus; ELISA: enzyme-linked immunosorbent assay; CMV: cytomegalovirus; EBV: Epstein-Barr virus; Anti-CCP: anti-cyclic citrullinated peptide; ANA: antinuclear antibody; AntidsDNA: anti-double-stranded DNA; ANCA: antineutrophil cytoplasmic antibodies

Electrocardiogram (ECG) and chest X-ray were unremarkable. Ultrasound abdomen was negative for hepatosplenomegaly, ascites, or fluid collection in the abdomen and pelvis. Echocardiography did not show any infective endocarditis. CT of the chest, abdomen, and pelvis with contrast revealed no enlarged lymph nodes or collections. Peripheral blood film did not reveal any blast cells, and bone marrow aspiration and biopsy showed healthy cellular bone marrow with trilineage hematopoiesis and no hemophagocytosis.

The initial impression was of bacterial infection, and the patient received broad-spectrum antibiotics for the appropriate duration; however, he continued to spike daily fever. He had an unremarkable ferritin level at presentation. After the initial workup virtually excluded TB, ongoing occult infection, lymphoma, and hematologic malignancy, ferritin was repeated. It was found that the patient exhibited marked hyperferritinemia (Figure 1).

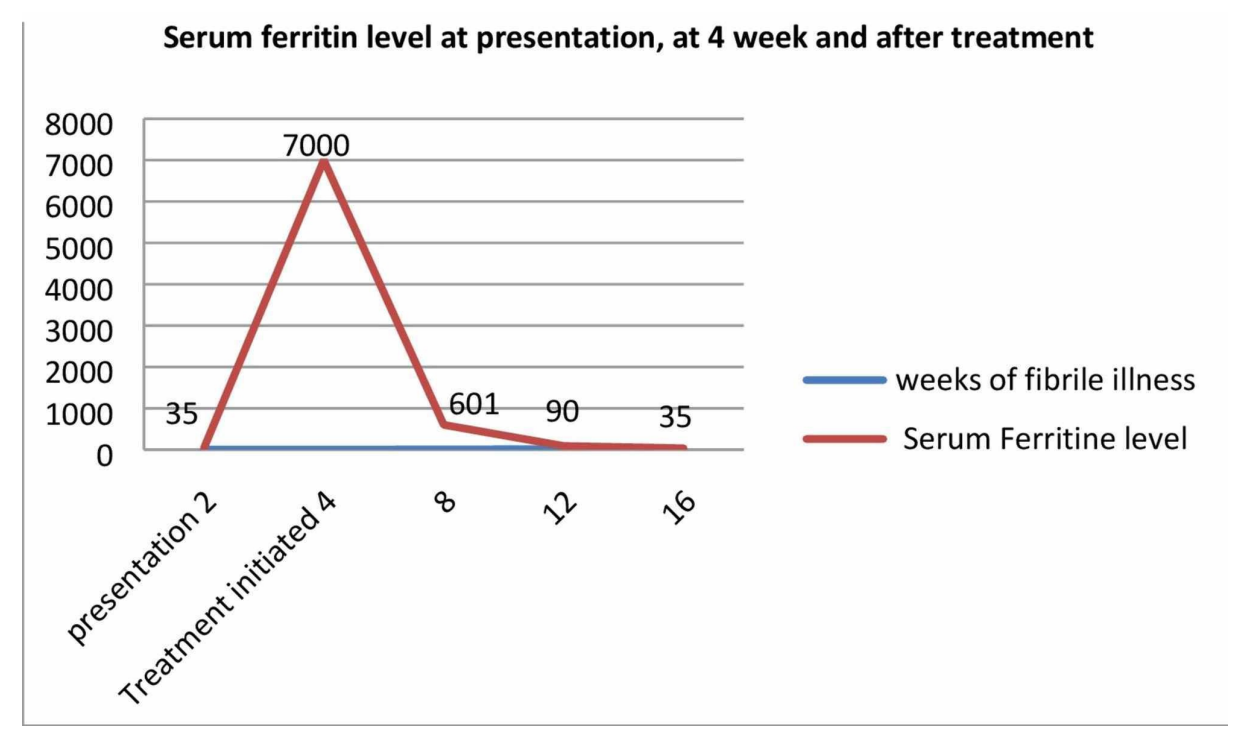


After excluding all plausible alternative diagnoses, ASD was diagnosed based on the Yamaguchi's criteria. The patient was treated with oral steroids. Consequently, his fever subsided, and he started gaining weight. Arthralgia subsided more gradually over time. Prednisolone was tapered down, and methotrexate was added to the treatment regimen. The patient responded well to the treatment. Subsequently, all his symptoms improved and serum ferritin levels normalized, and he has been symptom-free for almost a year now.

\section{Discussion}

The diagnosis of ASD is often delayed as it is a diagnosis of exclusion. The etiology and pathogenesis are still unclear, but some bacterial and viral infections and certain malignancies may act as inciting factors. No clear association between specific HLA loci or single genes and ASD has been established so far [6]. Specific cytokines may be involved in the pathogenesis of ASD. Serum levels of interleukin 6 (IL-6) and IL-18 have been found to exhibit a correlation with the clinical activity score of ASD [7].

Pyrexia of unknown origin (PUO) refers to a prolonged febrile illness without an established etiology despite extensive evaluation and diagnostic testing. Infections, non-infectious inflammatory etiology, and malignancies are common causes, and a significant number of patients remain undiagnosed [8]. ASD is the most common rheumatologic disorder presenting as PUO in young and middle-aged adults [9].

The most common symptoms of ASD are high-grade fever ( $39^{\circ} \mathrm{C}$ or above) in more than $95 \%$ of patients with one or more daily fever spikes. Also, large joint polyarthritis or arthralgia is also present in about $95 \%$ of cases as well as worsening joint pain during fever. The fusion of wrist joints is one of the specific radiological presentations [10]. The typical skin rash that appears in $80 \%$ of patients is salmon-colored, non-pruritic, non-scarring, and grows during fever spikes with positive Koebner phenomenon. Nonsuppurative pharyngitis, hepatomegaly, transaminitis, serositis, and rare involvement of renal, ophthalmic, and neurological systems have also been reported [11].

The disease presentation may be monophasic, intermittent, or chronic articular-type; each type roughly accounts for one-third of the cases. The type of presentation has an impact on prognosis [12]. Due to a lack of pathognomic clinical features and definitive diagnostic laboratory tests, ASD is usually diagnosed by applying the widely accepted Yamaguchi criteria. The presence of five features, with at least two of them being major criteria, is required for the diagnosis (Table 3) [13]. The Fautrel criteria have a higher specificity but lower sensitivity when compared to the Yamaguchi criteria; therefore, the latter is used more frequently to rule out ASD [14].

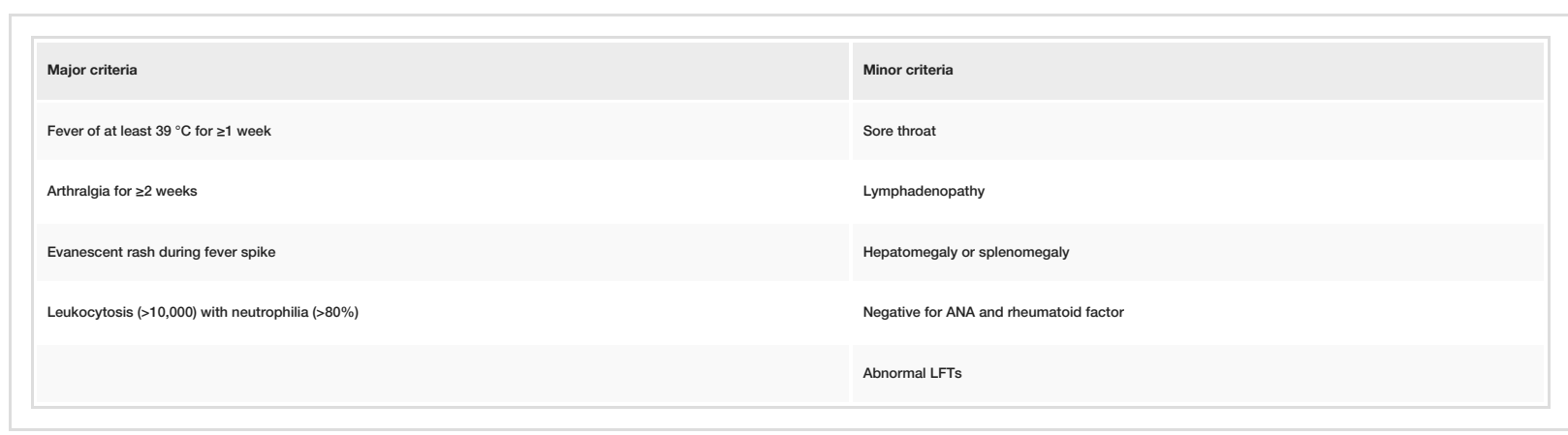

\section{TABLE 3: Yamaguchi criteria for the diagnosis of adult Still's disease}

Diagnosis requires the patients to have at least five features, with at least two of them being major diagnostic criteria

ANA: antinuclear antibody; LFT: liver function test

The characteristic laboratory findings are lymphocytosis with neutrophilia, normocytic normochromic anemia and thrombocytosis, high CRP, erythrocyte sedimentation rate (ESR), and transaminitis [15]. Marked hyperferritinemia is a common feature of ASD but is not pathognomonic. Elevated ferritin levels are associated with other rheumatological conditions. ASD is associated with a higher serum ferritin level of more than five times the upper limits of normal ( $>1,000 \mathrm{ug} / \mathrm{dl}$ ), sometimes ranging up to $>50,000 \mathrm{ug} / \mathrm{dl}$ [16]. Ferritin level also correlates with disease activity and is used as a biomarker to monitor therapeutic response [17]. The hypothesized mechanism involves cytokines-mediated ferritin synthesis. However, cases of ASD 
without a high ferritin level have been reported [18].

In our case, the serum ferritin level was lagging at the time of disease onset, which kept the suspicion of ASD low. The glycosylated ferritin level in ASD patients is lower $(<20 \%)$ than that of an average healthy person (50-80\%). The sensitivity and specificity of $<20 \%$ glycosylated ferritin in ASD are 78 and $64 \%$ respectively. A combination of the rise in serum ferritin and glycosylated ferritin levels has less sensitivity (43\%) but high specificity (93\%) for ASD [16].

The common treatment options available for ASD are non-steroidal anti-inflammatory drugs (NSAIDs), glucocorticoids, and disease-modifying anti-rheumatic drugs (DMARDs). The preferred treatment option for the articular disease is methotrexate; however, anakinra is preferred for systemic disease. Observational studies have suggested that DMARDs started within six months of the onset of chronic articular involvement may be associated with lower rates of joint injury, but prospective studies are needed to validate these observations [19]. Anti-IL-1 based therapies help to prevent the progression of amyloid [20]. Our patient had a moderate disease presentation. He was initially treated with oral steroids and later switched to methotrexate due to weight gain and persistent articular symptoms. The most severe complication of ASD is hemophagocytic lymphohistiocytosis (HLH), a syndrome resulting from excessive immune system activation.

\section{Conclusions}

ASD is a difficult disease to diagnose. It should be a significant consideration as a cause of PUO in young and middle-aged patients when rheumatologic, infectious, and malignant workups are negative. Atypical presentations may involve the absence of the typical skin rash and low serum ferritin levels in early disease. This phenomenon is rare and would be a diagnostic dilemma for the clinician, and may lead to underdiagnosis. The authors recommend that serum ferritin levels be repeated if PUO workup is unyielding despite an initial normal level. Early diagnosis and initiation of appropriate therapy can help to prevent complications and improve the prognosis for patients with ASD.

\section{Additional Information \\ Disclosures}

Human subjects: Consent was obtained by all participants in this study. Conflicts of interest: In compliance with the ICMJE uniform disclosure form, all authors declare the following: Payment/services info: All authors have declared that no financial support was received from any organization for the submitted work. Financial relationships: All authors have declared that they have no financial relationships at present or within the previous three years with any organizations that might have an interest in the submitted work. Other relationships: All authors have declared that there are no other relationships or activities that could appear to have influenced the submitted work.

\section{References}

1. Magadur-Joly G, Billaud E, Barrier JH, Pennec YL, Masson C, Renou P, Prost A: Epidemiology of adult Still's disease: estimate of the incidence by a retrospective study in west France. Ann Rheum Dis. 1995, 54:587-90. 10.1136/ard.54.7.587

2. Balci MA, Pamuk ÖN, Pamuk GE, Uzundere FK, Donmez S: Epidemiology and outcome of adult-onset Still's disease in Northwestern Thrace region in Turkey. Clin Exp Rheumatol. 2015, 33:818-23.

3. Bywaters EG: Still's disease in the adult. Ann Rheum Dis. 1971, 30:121-33. 10.1136/ard.30.2.121

4. Colebunders R, Stevens WJ, Vanagt E, Snoeck J: Adult Still's disease caused by Yersinia enterocolitica infection. Arch Intern Med. 1984, 144:1880-2. 10.1001/archinte.1984.00350210210040

5. Maset M, Costa EG, Carniello GS, Grazioli S, Ghersetti M, Casarin P: Fever and erythema: exclude all and then... think of Still's disease!. Italian J Med. 2016, 10:9. 10.4081/itjm.2016.680

6. Siddiqui M, Putman MS, Dua AB: Adult-onset Still's disease: current challenges and future prospects . Open Access Rheumatol. 2016, 8:17-22. 10.2147/OARRR.S83948

7. Chen DY, Lan JL, Lin FJ, Hsieh TY: Proinflammatory cytokine profiles in sera and pathological tissues of patients with active untreated adult onset Still's disease. J Rheumatol. 2004, 31:2189-98.

8. Efstathiou SP, Pefanis AV, Tsiakou AG, Skeva II, Tsioulos DI, Achimastos AD, Mountokalakis TD: Fever of unknown origin: discrimination between infectious and non-infectious causes. Eur J Intern Med. 2010, 21:137-43. 10.1016/j.ejim.2009.11.006

9. Arnow PM, Flaherty JP: Fever of unknown origin. Lancet. 1997, 350:575-80. 10.1016/S0140-6736(97)07061$\mathrm{X}$

10. Björkengren AG, Pathria MN, Sartoris DJ, Terkeltaub R, Esdaile JM, Weisman M, Resnick D: Carpal alterations in adult-onset Still disease, juvenile chronic arthritis, and adult-onset rheumatoid arthritis: comparative study. Radiology. 1987, 165:545-8.

11. Cozzi A, Papagrigoraki A, Biasi D, Colato C, Girolomoni G: Cutaneous manifestations of adult-onset Still's disease: a case report and review of literature. Clin Rheumatol. 2016, 35:1377-82. 10.1007/s10067-0142614-2

12. Yerra S, Tlhabano L, Vasamsetty T: Case report of atypical Still's disease: a diagnosis of exclusion . Int Med Case Rep J. 2016, 9:321-4. 10.2147/IMCRJ.S117664

13. Yamaguchi M, Ohta A, Tsunematsu T, et al.: Preliminary criteria for classification of adult Still's disease . J Rheumatol. 1992, 19:424-30. 


\section{Cureus}

14. Fautrel B, Zing E, Golmard JL, et al.: Proposal for a new set of classification criteria for adult-onset Still disease. Medicine (Baltimore). 2002, 81:194-200. 10.1097/00005792-200205000-00003

15. Kontzias A, Efthimiou P: Adult-onset Still's disease: pathogenesis, clinical manifestations and therapeutic advances. Drugs. 2008, 68:319-37. 10.2165/00003495-200868030-00005

16. Mehta B, Efthimiou P: Ferritin in adult-onset Still's disease: just a useful innocent bystander? . Int J Inflam. 2012, 2012:298405. 10.1155/2012/298405

17. Kirino Y, Kawaguchi Y, Tada Y, et al.: Beneficial use of serum ferritin and heme oxygenase-1 as biomarkers in adult-onset Still’s disease: a multicenter retrospective study. Mod Rheumatol. 2018, 28:858-64. 10.1080/14397595.2017.1422231

18. Omagari K, Matsunaga Y, Yamashita H, et al.: Successful treatment with cyclosporin in adult-onset Still disease manifesting as acute hepatitis with marked hyperferritinemia. Am J Med Sci. 2003, 326:148-51. 10.1097/00000441-200309000-00009

19. Franchini S, Dagna L, Salvo F, Aiello P, Baldissera E, Sabbadini MG: Efficacy of traditional and biologic agents in different clinical phenotypes of adult-onset Still's disease. Arthritis Rheum. 2010, 62:2530-5. 10.1002/art.27532

20. Topaloglu R, Batu ED, Orhan D, Ozen S, Besbas N: Anti-interleukin 1 treatment in secondary renal amyloidosis associated with autoinflammatory diseases. Pediatr Rheumatol Online J. 2015, 13:P149. 10.1186/1546-0096-13-S1-P149 